\title{
Molecular cloning and expression analysis of a stress-responsive WRKY transcription factor gene, BnWRKY57, from Brassica napus
}

\author{
Fatemeh Atashi Shirazi ${ }^{1}$, Hooman Razi ${ }^{2 *}$, Ali Niazi ${ }^{1}$, Abbas Alemzadeh $^{2}$ \\ ${ }^{1}$ Institute of Biotechnology, Shiraz University, Shiraz, Iran \\ ${ }^{2}$ Department of Crop Production and Plant Breeding, School of Agriculture, Shiraz University, Shiraz, Iran
}

*Corresponding author: razi@shirazu.ac.ir

\begin{abstract}
WRKY transcription factors play important roles in regulation of various plant biological processes, including response to abiotic stresses. WRKY genes might be potential targets for transgenic breeding to enhance stress tolerance in rapeseed (Brassica napus). The present study aimed to clone and characterize WRKY57 (BnWRKY57) gene derived from B. napus and to analyze patterns of BnWRKY57 expression under drought and salt stresses in two B. napus cultivars with different levels of tolerance to drought and salt. The full-length coding region of BnWRKY57 gene with $882 \mathrm{bp}$ long (GenBank Accession Number: MG699908) was cloned and sequenced. BnWRKY57 gene encodes a hydrophilic polypeptide of 293 amino acids. It shared high homology with other known WRKY57s from Brassicaceae family. The promoter of BnWRKY57 gene contained cis regulatory elements involved in response to phytohormones, light, biotic and abiotic stresses suggesting this gene may play a role to modulate different signaling pathways. Real time quantitative RT-PCR analysis revealed that $B n W R K Y 57$ expression was responsive to drought and salt stresses. BnWRKY57 gene showed different expression patterns between leaves and roots and also between the $B$. napus cultivars under stress conditions. Overall, the findings suggest that $B n W R K Y 57$ gene may contribute to adaptive responses to drought and salt stresses in $B$. napus.
\end{abstract}

Keywords: Rapeseed; WRKY57; Drought; Salt stress; Promoter analysis.

Abbreviations: ABRE_Abscisic acid Responsive Element; AP2/ERF_APETALA2/Ethylene Response Factor; bZIP_Basic Leucine Zipper; ChIP_Chromatin immunoprecipitation; CRE_Cis Regulatory Element; HSE_Heat-Shock Element; MYB_Myeloblastosis; MBS_MYB binding site.

\section{Introduction}

Drought and high salinity are critical environmental constraints that greatly limit plant growth and productivity. Plants utilize and coordinate a variety of molecular and cellular mechanisms to respond and cope with these harsh stresses. Transcriptional regulation is a fundamental process to control expression of genes involved in plant responses to environmental stresses. Identification and characterization of stress-responsive regulatory genes have revealed potential target genes to enhance plant stress tolerance through genetic engineering (Yang et al., 2010). Transcription factors are key components of cell regulatory machinery, as they act to modulate transcription of numerous downstream genes involved in diverse plant processes. Various families of transcription factors such as AP2/ERF, bZIP, MYB and WRKY significantly contribute to plant stress response and tolerance (YamaguchiShinozaki and Shinozaki, 2006; Ren et al., 2010).

WRKY transcription factors are a large family of plant transcriptional regulators containing one or two WRKY domain with approximately 60 amino acids long (Eulgem et al., 2000).
WRKY domain is characterized by a highly conserved amino acid motif, WRKYGQK, at N-terminus together with a zincfinger like motif at C terminus (Rushton et al., 2010). Arabidopsis WRKY proteins are classified into three groups based on number of WRKY motifs and features of Zinc-finger like motif (Eulgem et al., 2000). WRKY domain generally binds to $\mathrm{W}$-box elements containing $\operatorname{TTGAC}(\mathrm{C} / \mathrm{T})$ motif, present in promoter regions of target genes (Fan et al., 2015; Rinerson et al., 2015).

Plant WRKY transcription factors are known to function in various physiological processes, such as trichome development (Johnson et al., 2002; Huang et al., 2015), embryogenesis (Lagacé and Matton, 2004; Huang et al., 2015), seed development (Luo et al., 2005; Huang et al., 2015), dormancy (Pnueli et al., 2002; Huang et al., 2015), and senescence (Hinderhofer et al., 2001; Huang et al., 2015). Furthermore, previous studies have demonstrated essential roles of WRKYs in plant responses to internal and external stimuli. WRKY proteins are involved in plant defence responses against 
bacterial, fungal, and viral pathogens (Eulgem and Somssich, 2007; Pandey et al., 2009; Huang et al., 2015; Jiang and Yu, 2016). They are also key components in a number of signal transduction processes mediated by plant hormones such as gibberellic acid (Zhang et al., 2004; Huang et al., 2015), abscisic acid (ABA) (Rushton et al., 2012; Huang et al., 2015), and salicylic acid (Du and Chen, 2000; Huang et al., 2015). For instance, Arabidopsis WRKY57 acts as a regulatory node in Jasmonic acid (JA)-induced senescence signalling pathways mediated by auxin and JA (Jiang et al., 2014).

It is also well documented that WRKY proteins are involved in response to abiotic stresses such as salinity, drought, and cold (Zhou et al., 2008; Ren et al., 2010; Huang et al., 2015). Several Arabidopsis WRKY genes are induced by environmental stresses (Seki et al., 2007; Ma et al., 2014; He et al., 2016). Previous findings indicated that AtWRKY46 modulates osmotic stress responses and stomatal movement in Arabidopsis (Ding et al., 2014; He et al., 2016). In addition, Arabidopsis WRKY63/ABO3 regulates responses to $A B A$ and drought tolerance (Ren et al., 2010). Overexpression studies further supported involvement of WRKYs in conferring tolerance to abiotic stresses. Overexpression of the stress-induced OsWRKY45 improved drought tolerance in Arabidopsis (Qiu and $Y u, 2009)$. Transgenic Arabidopsis plants Overexpressing TaWRKY2 or TaWRKY19 from wheat showed enhanced tolerance to drought and salt stresses (Niu et al., 2012). Moreover, overexpression of Chrysanthemum genes DgWRKY1 and DgWRKY3 increased tolerance to salt stress in tobacco (Liu et al., 2013; Liu et al., 2014).

Previous reports have demonstrated the role of Arabidopsis WRKY57 in abiotic stress tolerance. Activated expression of WRKY57 enhanced drought tolerance of Arabidopsis by increasing $A B A$ levels and by positively regulating the expression of stress-responsive genes (Jiang et al., 2012). ChIP assays illustrated that Arabidopsis WRKY57 could directly bind the W-box within the promoter sequences of the stressresponsive genes, RD29A and NCED3 (Jiang et al., 2012). Transgenic rice (Oryza sativa) plants harbouring Arabidopsis WRKY57 exhibited improved tolerance to drought and salt stresses, indicating potential role of WRKY57 in crop improvement (Jiang et al., 2016).

Rapeseed (Brassica napus) is one of the world's leading oilseed crops. Rapeseed production is substantially affected by adverse environmental factors (Diepenbrock, 2000; Raymer, 2002; Xie et al., 2007). Development of rapeseed cultivars with enhanced tolerance to environmental stresses is, therefore, of great importance in rapeseed breeding programs. Knowledge of molecular regulatory mechanisms involved in abiotic stress responses may potentially be used for molecular breeding toward improved stress tolerance in rapeseed. WRKY genes might be potential targets for molecular breeding of Brassica crops against abiotic and biotic stresses. Despite the importance of WRKYs in response to abiotic stresses, there are few reports describing the roles of $B$. napus WRKY transcription factors in mediating responses to stresses. 13 $B n W R K Y$ genes have been identified that are responsive to both fungal pathogens and hormone treatments (Yang et al., 2009). Furthermore, the expression of 26 BnWRKY genes under multiple stresses revealed that most of the genes were induced by drought, salinity and low temperature (He et al., 2016).

To date, a comprehensive view of $B$. napus WRKY57 (BnWRKY57) is still lacking. The present study aimed to perform structural and functional characterization of BnWRKY57 gene. The coding sequence of BnWRKY57 was isolated, cloned and sequenced for the first time. Afterwards, the expression of BnWRKY57 gene in response to drought and salt stresses was analyzed in two $B$. napus cultivars with different levels of tolerance to drought and salt. Finally, the promoter region of BnWRKY57 was analyzed to identify putative cis-regulatory elements that may be involved in regulation of BnWRKY57 transcription.

\section{Results}

\section{Molecular cloning and characterization of BnWRKY57 gene}

The full-length coding sequence of BnWRKY57 gene was amplified using the specific primers. A single PCR product of expected size (around 900bp) was obtained and then cloned followed by sequencing. BnWRKY57 coding sequence (GenBank Accession Number: MG699908) was 882bp in length encoding a deduced protein of 293 amino acid residues with a predicted molecular weight of $32.552 \mathrm{kDa}$ and isoelectric point of 6.26. The BnWRKY57 deduced protein contained a single WRKY DNA binding domain (from $151^{\text {st }}$ to $208^{\text {th }}$ amino acid residue) indicating that BnWRKY57 protein is a member of WRKY transcription factor family (Fig. 1A). SOPMA analysis showed that the overall folding of BnWRKY57 protein is basically built with alpha helix (25.26\%) and random coils (60.07\%) that are connected with extended strands (10.92\%) and $\beta$-turns (3.75\%) (Fig. 1B). Hydropathicity analysis revealed that BnWRKY57 contained many hydrophilic amino acids suggesting that it is a hydrophilic protein (Fig. 2). According to a web bioinformatic tool, Distill, BnWRKY57 protein was localized in nucleus, indicating it plays regulatory roles mainly in cell nucleus.

BnWRKY57 shared highest homology (94\%) with Brassica rapa WRKY57 at the nucleotide level. In addition, BnWRKY57 deduced protein showed high amino acid sequence identity with WRKY57 proteins from other Brassica species including Brassica rapa (91.9\%), Brassica campestris (91.3\%) and Brassica oleracea (89.6\%).

The genomic structure of BnWRKY57 gene, similar to its Arabidopsis counterpart, contained three exons with sizes of 368, 141, and 373 nucleotides, respectively (Fig. 3). The structural analysis revealed that BnWRKY57 gene is located in the chromosome 7 of $B$. napus $A$ genome originated from its progenitor, $B$. rapa. Synteny analysis showed that $B$. rapa and $B$. oleracea, the putative donors of $B$. napus $A$ and $C$ genomes, have orthologs of BnWRKY57 located in the chromosomes 7 and 6 of their genomes, respectively.

A phylogenetic tree was reconstructed based on the amino acid sequences deduced from WRKY57 genes from various plant species (Fig. 4). The results showed that WRKY57 genes from species of Brassicaceae family were grouped together supported by a high bootstrap value. Indeed, WRKY57 genes of 


\title{
(A) IRKY57 amino acid sequence
}

\begin{abstract}
MTDPEDPDLSNEDSAWRQLTAPDSVFFNRDTSS ILSDFGWDLHASSSDNHHNLRFDPCLP PTSTVPSSVTTTATPDPIPASSHSSTVAAATASVVSTSNNPSATSSSSEGPTDNSTPKTP ETPKKEKKQAQKRIRQPRFAFMTKSDVDNLEDGYRWRKYGQKAVKNSSFPRSYYRCTNSR CTVKKRVERSSEDPS IVITTYEGQHCHQTVGFPRGGIFPAHDPHNFTSHHHLPPPLPNPY YYQELLHQLHRE ITS S PQLPQSTTDDGHFAVPS INQPEEGLLGDIVPQTMRNP
\end{abstract}

(B)

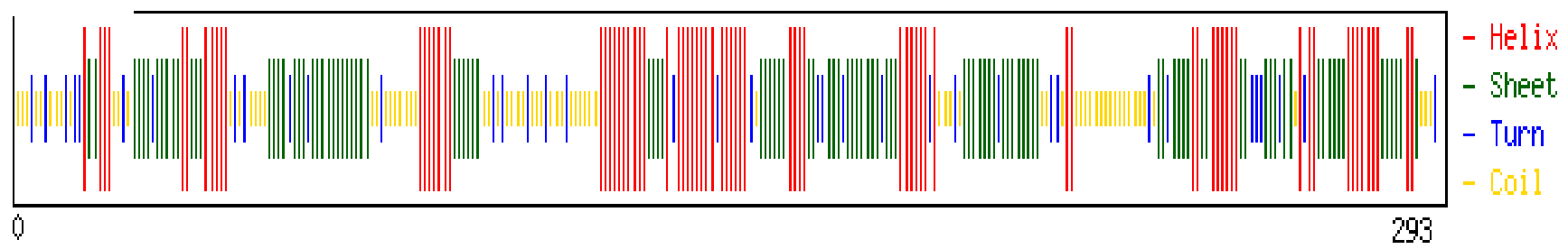

Fig 1. (A) The deduced protein of BnWRKY57 gene containing 293 amino acids. The highlighted amino acid sequence (from $151^{\text {st }}$ to $208^{\text {th }}$ amino acid residue) represents a WRKY DNA binding domain. (B) The secondary structure of BnWRKY57 protein. Alpha helix, extended strand, beta turn and random coil are shown in red, green, blue and yellow vertical lines, respectively.

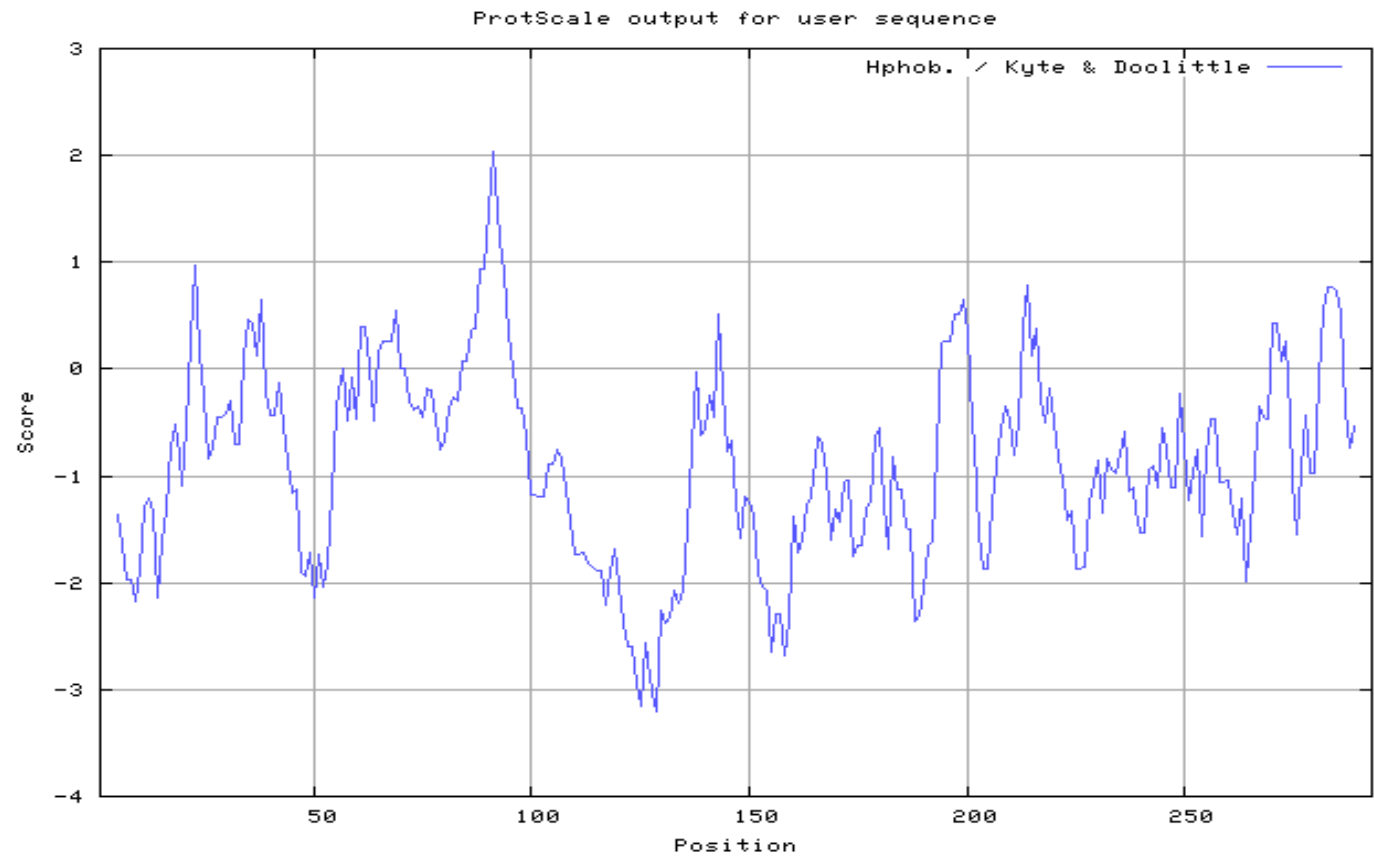

Fig 2. Hydropathicity plot for BnWRKY57 protein according to Kyte and Doolittle method. Amino acid positions are presented on the Xaxis. The hydropathicity scores for individual amino acids are on the $Y$-axis. 


\begin{tabular}{|c|c|c|c|c|}
\hline $368 \mathrm{bp}$ & $768 \mathrm{bp}$ & 141bp & 139bp & 373 bp \\
\hline
\end{tabular}

Fig 3. Schematic diagram of the genomic structure of BnWRKY57 gene. Exons and introns are shown in blue and red boxes, respectively.

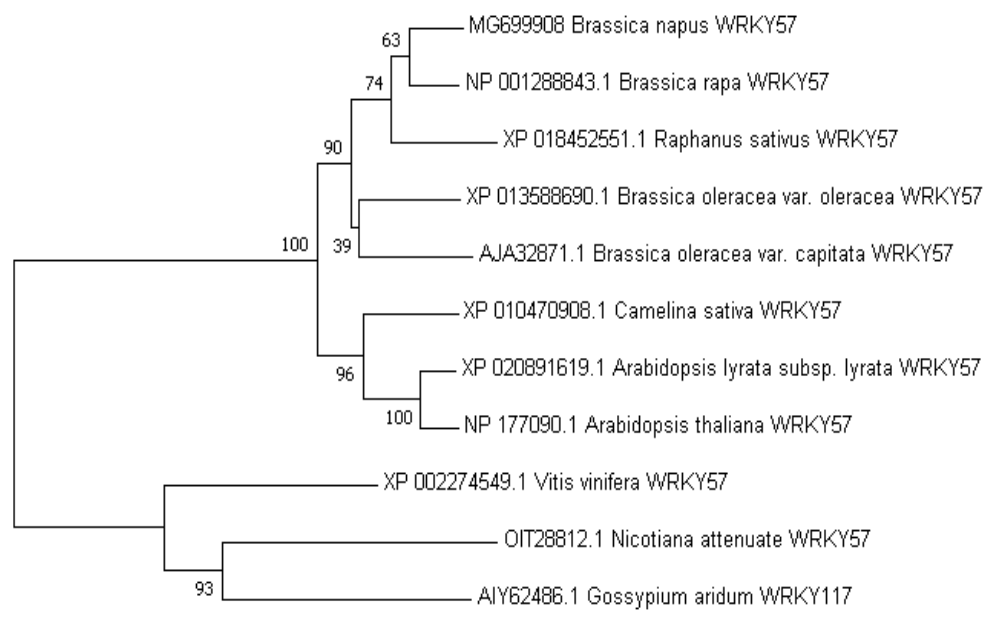

$\stackrel{\vdash}{0.050}$

Fig 4. Neighbour-joining phylogenetic tree based on aligned amino acid sequences deduced from WRKY57 genes from Brassicaceae family and other plant species. GenBank accession numbers of individual genes are shown in the phylogenetic tree. Values at the nodes indicate bootstrap support (\%) out of 1000 replicates.

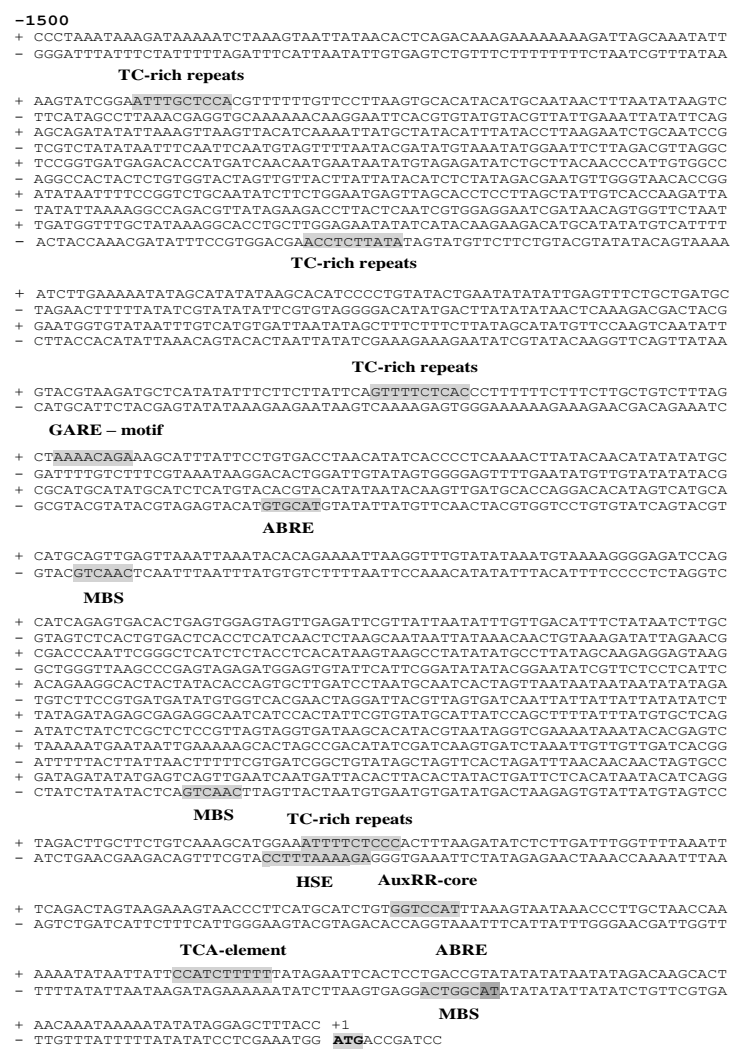

Fig 5. 1500 bp upstream of the start codon of BnWRKY57 gene. The putative CREs involved in response to phytohormones and abiotic stresses are shown in the promoter region. 

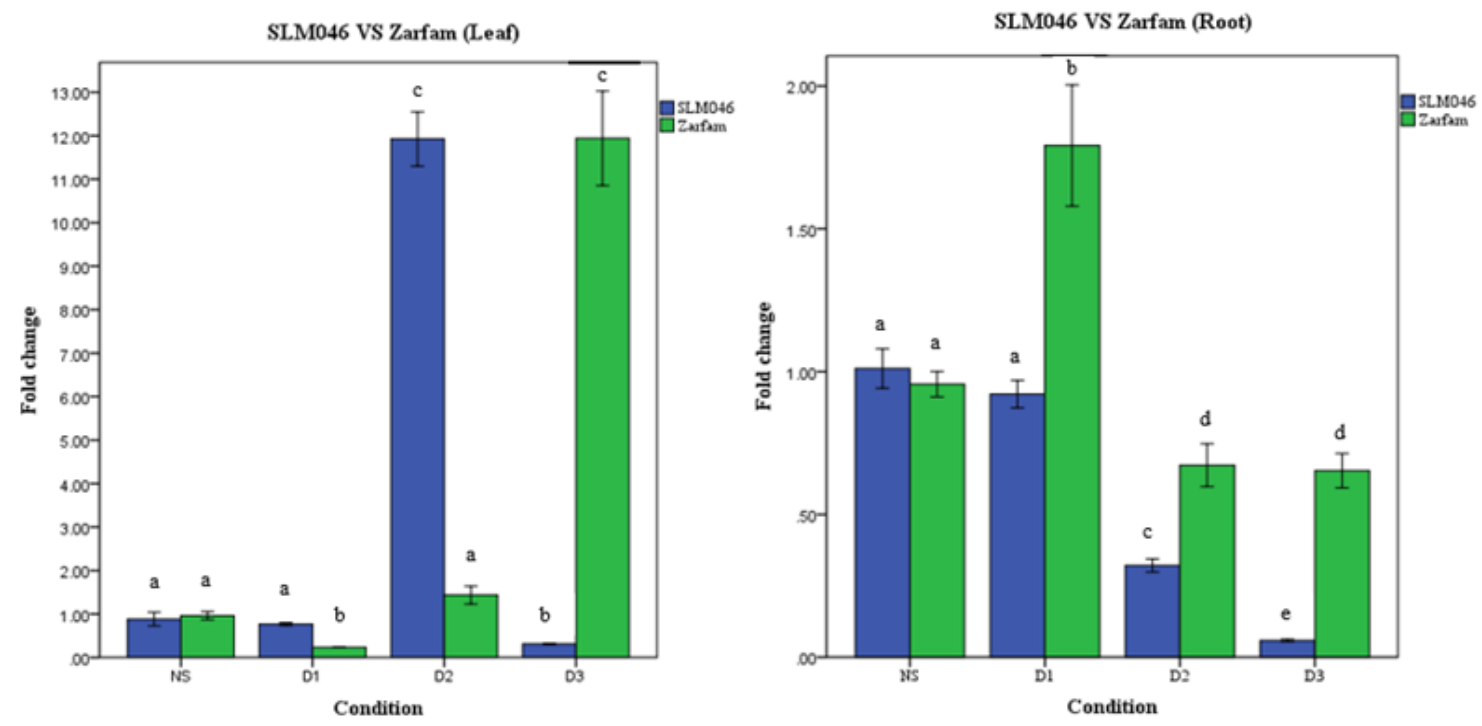

Fig 6. Expression of BnWRKY57 gene in B. napus cultivars (SLM046 and Zarfam) under drought stress. A: BnWRKY57 expression in leaves. B: BnWRKY57 expression in roots. The expression data were determined by real time quantitative RT-PCR. The drought stress treatments include the time that soil moisture reached to $40 \%$ of field capacity (D1) as well as maintaining that for $6 \mathrm{~h}$ (D2) and $24 \mathrm{~h}$ (D3). NS denotes non-stress condition. Different letters indicate significant differences according to LSD test $(P<0.05)$.
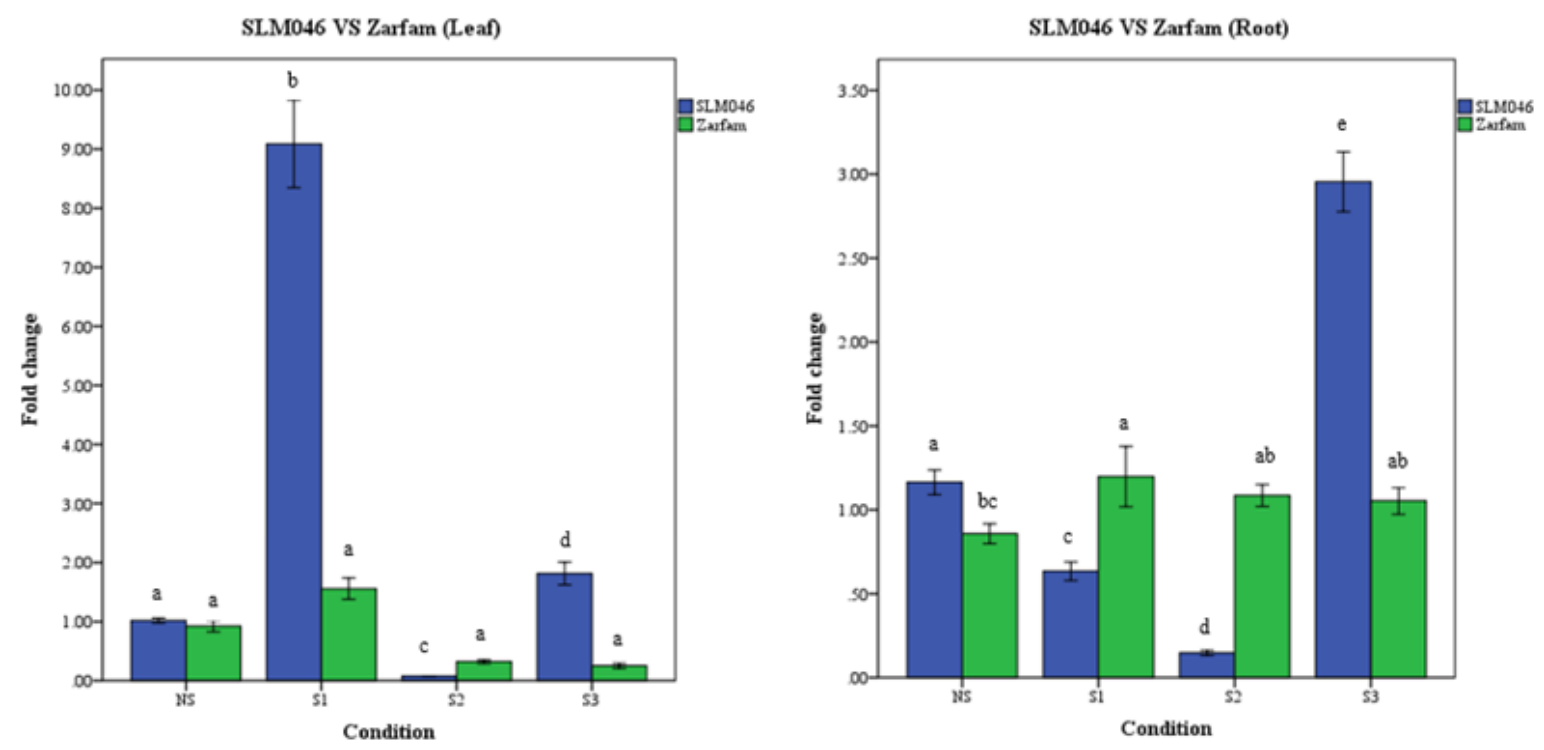

Fig 7. The expression of BnWRKY57 gene in B. napus cultivars (SLM046 and Zarfam) under salt stress. A: BnWRKY57 expression in leaves. B: BnWRKY57 expression in roots. The expression data were determined by real time quantitative RT-PCR. The salt stress treatments include $3 \mathrm{~h}$ (S1), $24 \mathrm{~h}$ (S2) and 7 days (S3) after adding salt solution. NS denotes non-stress condition. Different letters indicate significant differences according to LSD test $(P<0.05)$. 
Table 1. Cis regulatory elements (CREs) identified in the promoter region of BnWRKY57 gene.

\begin{tabular}{|c|c|c|}
\hline CRE & Function & Number of occurrences \\
\hline 3-AF1 binding site & Light responsive element & 1 \\
\hline A-box & Regulatory element & 1 \\
\hline AAGAA-motif & Unknown & 2 \\
\hline ABRE & Abscisic acid responsive element & 2 \\
\hline ACE & Light responsive element & 1 \\
\hline ARE & Anaerobic inducible element & 2 \\
\hline AuxRR-core & Auxin responsive element & 1 \\
\hline Box 4 & Light responsive element & 2 \\
\hline Box III & Protein binding site & 1 \\
\hline CAAT-box & Cis-acting elements /enhancer & 25 \\
\hline CCGTCC-box & Meristem specific activation element & 1 \\
\hline G-box & Light responsive element & 3 \\
\hline GAG-motif & Light responsive element & 1 \\
\hline GARE - motif & Gibberellin responsive element & 1 \\
\hline GCN4-motif & endogenous expression element & 1 \\
\hline HSE & Heat stress responsive element & 1 \\
\hline I-box & Light responsive element & 1 \\
\hline MBS & Drought inducible element & 3 \\
\hline Skn-1-motif & Endosperm expression element & 3 \\
\hline TATA-box & Core promoter element & 32 \\
\hline TCA-element & Salicylic acid responsive element & 1 \\
\hline TCT- motif & Light responsive element & 3 \\
\hline As-2-box & $\begin{array}{l}\text { shoot-specific expression and Light } \\
\text { responsive element }\end{array}$ & 1 \\
\hline Chs-CMA2a & Light responsive element & 1 \\
\hline
\end{tabular}


Table 2. List of primers used for isolation and expression analysis of BnWRKY57 and Bnelf1 $\alpha$ genes.

\begin{tabular}{llc}
\hline Gene & Primer & $\begin{array}{c}\text { Amplicon length on } \\
\text { CDNA(bp) }\end{array}$ \\
\hline \multirow{2}{*}{ BnWRKY57 } & BWRKY-F 5'-ATGACCGATCCTGAAGATCCC-3' & 882 \\
\multirow{2}{*}{ BnWRKY57 } & BWRKY-R 5'-GGAGCTTTACCTCAAGGATTGC-3' \\
\multirow{2}{*}{ Bnelf1 $\alpha$} & BWRT-F 5'-GCTACTGCTTCTGTTGTCTCG-3' & 121 \\
& BWRT-R 5'-CCTGCTTCTTCTCCTTCTTTGG-3' & \\
& Elf-F 5'-AGCCGCAAGTCCTCCTCTCAG-3' & 186 \\
\hline
\end{tabular}

Brassica species formed a monophyletic group suggesting they have been probably originated from a common ancestral gene. Moreover, WRKY57 genes of Brassica species fell into a subgroup separated from their Arabidopsis orthologs. The phylogenetic reconstruction also uncovered that BnWRKY57 was most closely related to B. rapa WRKY57.

\section{Analysis of BnWRKY57 promoter}

The $1.5 \mathrm{~kb}$ upstream promoter region of BnWRKY57 gene was analyzed using PlantCARE database to identify putative CREs. A total of 95 CREs from 25 different types were identified within the BnWRKY57 promoter sequence (Table 1). The positions of some CREs are shown in Fig. 5. BnWRKY57 promoter contained several hormone-responsive CREs associated with activity and regulation of abscisic acid, gibberellin, auxin and salicylic acid implying that BnWRKY57 may be involved in hormone crosstalk. Moreover, a number of putative CREs involved in tissue-specificity, defence, light response and anaerobic induction were found in the BnWRKY57 promoter. The CREs related to responses to heat and drought stresses (HSE and MBS motifs) were also detected in the BnWRKY57 promoter, indicating that the gene is involved in plant responses to environmental stresses.

\section{Expression pattern of BnWRKY57 under drought stress}

The expression pattern of BnWRKY57 was examined in response to drought stress using real time quantitative RT-PCR (qRT-PCR) in leaves and roots of the $B$. napus cultivars. The different expression profiles were observed for the gene not only between the cultivars but also between leaves and roots during drought stress period. In leaves of the drought tolerant cultivar (SLM046), BnWRKY57 expression was more quickly induced in response to drought stress and showed the maximum level of transcript accumulation at 6 hours after maintaining soil moisture at $40 \%$ of field capacity (D2) (Fig. 6A). Moreover, BnWRKY57 expression exhibited a significantly higher level of induction in leaves of the drought tolerant cultivar, SLM046, compared to that of the other B. napus cultivar (Zarfam). BnWRKY57 expression was significantly downregulated in roots of SLM046 during drought stress period, whilst it significantly increased in roots of Zarfam at the first drought stress treatment (D1) (Fig. 6B) and then showed a significant downregulation at 6 (D2) and 24 (D3) hours after maintaining soil moisture at $40 \%$ of field capacity.

\section{Expression pattern of BnWRKY57 under salt stress}

The expression pattern of BnWRKY57 was analysed under salt stress condition using qRT-PCR in leaves and roots of the $B$. napus cultivars. The results revealed significant differences for the expression of BnWRKY57 between the cultivars and also between leaf and root tissues. BnWRKY57 expression rapidly and sharply increased in leaves of the $B$. napus salt tolerant cultivar, SLM046, and reached its maximum level at three hours following salt stress (S1) in which the transcript accumulation of BnWRKY57 was almost nine times higher than that of non-stress conditions (Fig. 7A). BnWRKY57 expression was then significantly downregulated and showed the minimum transcript level at 24 hours (S2) after salt stress application. BnWRKY57 expression was also induced in leaves of the $B$. napus salt sensitive cultivar, Zarfam, at S1, however much less induction occurred in comparison with that of SLM046 (Fig. 7A). BnWRKY57 expression significantly declined in roots of SLMO46 at 3 (S1) and 24 (S2) hours following salt stress, and then showed a dramatic upregulation as the transcript accumulation of BnWRKY57 exhibited an almost 3fold increase at 7 days post salt stress (S3) compared to nonstress conditions (Fig. 7B). On the other hand, BnWRKY57 expression was slightly induced in roots of Zarfam by salt stress, and showed no significant changes during salt stress (Fig. 7B).

\section{Discussion}

WRKY genes constitute a large and important gene family in higher plants. Accurate annotation of structure and function of these transcription factor genes is an essential step for further in-depth understanding plant regulatory pathways. In the present study, we isolated, cloned and sequenced the coding region of $B$. napus WRKY57 encoding a protein of 293 amino acids. BnWRKY57 exhibited a high degree of similarity with WRKY57 proteins from Arabidopsis and Brassica species implying they may have conserved functions. Increasing evidence suggests that WRKY genes are involved in various biological processes, including plant development, and responses to biotic and abiotic stresses (Eulgem and Somssich, 2007; Rushton et al., 2010; Luo et al., 2013; Liu et al., 2016; Zhao et al., 2015). Phylogenetic relationships revealed that WRKY57 genes from diploid Brassica species ( $B$. rapa and $B$. oleracea) and the amphidiploid $B$. napus fell into the same clade supporting the view reported by Rana et al. (2004) that gene duplication events occurred before the divergence of the diploid Brassica species. 
The promoter analysis suggested that BnWRKY57 gene expression is regulated by modulators, such as phytohormones, light, drought. In addition, BnWRKY57 gene with diversified CREs may play as a mediator to link different signalling pathways; for instance, between phytohormones and abiotic stress signalling pathways. Plant hormones, as essential endogenous signal molecules, can regulate cellular processes, plant growth, and development under stress conditions (Grove et al., 1979; Kermode, 2005; Ryu and Cho, 2015). Previous findings indicated that the expression of WRKY genes is affected by hormone treatments (Yang et al., 2009; Jiang et al., 2014). The expression analysis showed that BnWRKY57 gene was significantly induced by drought stress. The different expression patterns of BnWRKY57 gene between the cultivars during drought stress were somewhat associated with the level of drought tolerance of the cultivars. Accordingly, a strong and quick upregulation of BnWRKY57 expression was observed in leaves of the drought tolerant cultivar (SLM046) compared to the drought sensitive cultivar (Zarfam). Similar to BnWRKY57, B. rapa WRKY57 was upregulated up to 4 hours after drought stress treatment and then showed downregulation (Abdul Kayum et al., 2015). Arabidopsis WRKY57 in transgenic rice plants enhanced tolerance to drought and salt stresses (Jiang et al., 2016). It has been previously reported that activated expression of Arabidopsis WRKY57 enhanced adaption to drought tolerance by elevation of ABA levels that is closely involved in drought stress responses and by positively regulating the expression of stress-responsive genes (Jiang et al., 2012). Interestingly, the promoter region of BnWRKY57 contained ABRE motif that is involved in $A B A$ responsiveness and in early response to abiotic stresses leading to activate a broad range of adaptation mechanisms (Corrêa et al., 2008). Furthermore, the presence of HSE and MBS motifs in the BnWRKY57 promoter was in accordance with the responsiveness of BnWRKY57 expression to abiotic stresses.

BnWRKY57 gene was also induced by salt stress, however it showed different expression profiles between the salt tolerant and sensitive $B$. napus cultivars. This finding was in consistent with the expression pattern of $B$. rapa WRKY57 in response to salt stress (Abdul Kayum et al., 2015). Additionally, the expression analysis of $26 B n W R K Y$ genes uncovered that most of the genes responded to drought and salt stresses (He et al., 2016). It has already reported that WRKY57 may promote tolerance to osmotic and salt stresses in Arabidopsis by acting as a positive regulator in response to hyperosmotic stress (Jiang et al., 2012). Indeed, this study has provided evidence implying that BnWRKY57 gene may be involved in adaptive responses to abiotic stresses in $B$. napus, although it is essential to perform overexpression and knockout experiments to resolve the direct involvement of this gene in abiotic stress tolerance mechanisms.

\section{Materials and Methods}

\section{Cloning and sequencing of B. napus WRKY57 gene}

Total RNA was extracted from leaves of $B$. napus cv. SLM046 using column RNA isolation kit (DENA Zist Asia, Iran) according to manufacturer's instruction. RNA quantity and integrity were checked by agarose gel electrophoresis and spectrophotometry. DNA contamination was removed from RNA using Fermentas DNasel treatment (ThermoFisher Scientific, USA). First strand cDNA was synthesized from total RNA using Fermentas First Strand cDNA Synthesis Kit (ThermoFisher Scientific, USA). The full-length coding sequence of BnWRKY57 was amplified by PCR using the primers BWRKYF and BWRKYR (Table 2). The primers were designed based on a B. napus ortholog of WRKY57 identified by searching $B$. napus genome sequence (http://www.genoscope.cns.fr/spip/) against Arabidopsis WRKY57.

The PCR conditions were as follows: initial denaturation at $95^{\circ} \mathrm{C}$ for $5 \mathrm{~min}$, followed by 35 cycles of $95^{\circ} \mathrm{C}$ for $30 \mathrm{~s}, 60^{\circ} \mathrm{C}$ for $30 \mathrm{~s}$, $72^{\circ} \mathrm{C}$ for $90 \mathrm{~s}$; and finally, $72{ }^{\circ} \mathrm{C}$ for $10 \mathrm{~min}$. The PCR product was analysed by agarose gel electrophoresis $(1 \% \mathrm{w} / \mathrm{v})$. Subsequently, the PCR product was purified and cloned into pTZ57R/T vector (InsTAclone PCR Cloning kit, ThermoFisher Scientific, USA). Transformation of competent cells of E. coli strain DH5 $\alpha$ was performed by heat-shock method (Froger and Hall, 2007). Finally, the cloned PCR fragment was sequenced. The coding sequence of BnWRKY57 was submitted in GenBank under the accession number MG699908.

\section{In Silico sequence analysis}

The coding sequence of BnWRKY57 was subjected to similarity searches against non-redundant nucleotide and protein databases using BLASTN and BLASTX (E-value $\leq 10^{-5}$ ), respectively, available at NCBI website (http://www.ncbi.nlm.nih.gov/blast). The exon-intron structure and synteny of BnWRKY57 were determined using Blat tool of GenoScope (http://www.genoscope.cns.fr/spip/), the $B$. napus genome sequence database. The amino acid sequence of BnWRKY57 was deduced and the corresponding primary structure parameters such as molecular weight and isoelectric point were obtained using the ExPasy website (https://www.expasy.org/tools/). Hydropathicity of the deduced BnWRKY57 protein was computed by ProtScale (https://web.expasy.org/protscale/) according to Kyte and Doolittle method (Kyte and Doolittle, 1982). Secondary structure of BnWRKY57 was analysed using SOPMA tool (https://npsa-prabi.ibcp.fr/cgi-bin/secpred_sopma.pl). BnWRKY57 subcellular localization was predicted by Distill (http://distill.ucd.ie/distill/). Pfam (http://pfam.xfam.org/) (Finn et al., 2016) was used to identify functional domains within the BnWRKY57 protein.

Phylogenetic analysis was performed based on the amino acid sequences deduced from WRKY57 genes from Brassicaceae family and other plant species using neighbour-joining method with 1000 bootstrap replicates implemented by MEGA software Version 7 (Kumar et al., 2016). To characterize the promoter region of BnWRKY57 gene, $1500 \mathrm{bp}$ upstream of the start codon, as a putative promoter region, was obtained from GenoScope (http://www.genoscope.cns.fr/spip/). The promoter sequence was searched for cis-regulatory elements (CREs) using PlantCARE 
(http://bioinformatics.psb.ugent.be/webtools/plantcare/html/ ) (Lescot et al., 2002).

\section{Expression profiling of BnWRKY57 under drought and salt stress conditions}

The B. napus cultivars, SLM046 and Zarfam, with distinct responses to abiotic stresses (Rameeh et al., 2012; Darjani et al., 2013; Mirzaee et al., 2013; Shamloo-Dashtpagerdi et al., 2018), were employed to perform drought and salt stress experiments. The drought stress experiment was performed to analyse expression changes of BnWRKY57. Plants were grown in plastic pots filled with sterilized sandy-clay soil under growth room conditions (16h light/8h dark; $24^{\circ} \mathrm{C}$ day $/ 8^{\circ} \mathrm{C}$ night). Plants with five true leaves were exposed to drought stress by withholding water to reach soil moisture to $40 \%$ of field capacity (FC). Leaf and root samples were collected under nonstress together with three drought stress treatments including when soil moisture reached to $40 \%$ of FC as well as maintaining that for 6 and 24 hours. The non-stress condition was conducted by regularly irrigating plants to keep soil moisture around FC during the experiment.

The salt stress experiment was also conducted to assay expression levels of BnWRKY57. Surface-sterilized seeds of the $B$. napus cultivars were sown in sandy soil under a long-day photoperiod (16 h light/ $8 \mathrm{~h}$ dark) with 24 and 8 ㅇ C day and night temperature, respectively. 10-day-old seedlings were then transferred to a hydroponic system supplemented with half-strength Hoagland's nutrient solution $(\mathrm{pH} \approx 6.0)$ under growth room conditions. Salt stress was applied to plants with five true leaves by adding $150 \mathrm{mM} \mathrm{NaCl}$ solution to the medium. Samples of leaves and roots were collected under non-stress as well as three salt stress treatments including 3 hours, 24 hours and 7 days after adding salt solution.

qRT-PCR was used to assess mRNA expression levels of $B n W R K Y 57$ in leaves and roots of the $B$. napus cultivars during drought and salt stresses. Total RNA extraction and CDNA synthesis were done as described earlier for isolation of BnWRKY57. qRT-PCR was performed on an ABI StepOne realtime PCR machine (Applied Biosystems, USA) by using RealQ PCR Master Mix kit (Ampliqon, Denmark) according to the manufacturer's instructions. The thermal cycling conditions were as follows: $15 \mathrm{~min}$ at $95^{\circ} \mathrm{C}$, followed by 40 cycles at $95^{\circ} \mathrm{C}$ for $10 \mathrm{~s}, 60{ }^{\circ} \mathrm{C}$ for $15 \mathrm{~s}$, and $72{ }^{\circ} \mathrm{C}$ for $30 \mathrm{~s}$. qRT-PCR reactions were also performed for the reference gene, $B$. napus Elongation Factor $1 \alpha$ (Bnelf1 $\alpha$; GenBank Accession number: FJ529181) for normalization of expression data. The specific primers for BnWRKY57 (BWRTF and BWRTF) and Bnelf1 $\alpha$ are listed in Table 2. Three independent biological samples were used to do qRT-PCR. Each reaction was also repeated two times as technical replicates. The relative expression levels of BnWRKY57 gene were calculated using the $2^{-\Delta \Delta C T}$ method (Livak and Schmittgen, 2001). LSD statistical test was performed to determine significant differences between the expression levels.

\section{Conclusion}

This study is the first report on cloning and molecular characterization of the full-length coding sequence of $B$. napus transcription factor gene, BnWRKY57, aimed at providing a framework for future studies regarding BnWRKY57 functions. The expression patterns of BnWRKY57 gene of the two $B$. napus cultivars under drought and salt stress conditions along with analysis of the BnWRKY57 promoter suggested that this gene may serve as a regulator involved in responses to drought and salt stresses. Further in-depth studies are required to decipher the roles of BnWRKY57 gene in stress regulatory pathways.

\section{Acknowledgments}

The authors would like to thank Institute of Biotechnology of Shiraz University for financial and technical supports.

\section{References}

Abdul Kayum MD, Jung HJ, Park JI, Ahmed NU, Saha G, Yang TJ, Nou IS (2015) Identification and expression analysis of WRKY family genes under biotic and abiotic stresses in Brassica rapa. Mol Genet Genomics. 290:79-95.

Corrêa LGG, Riaño-Pachón DM, Schrago CG, Dos Santos RV, Mueller-Roeber B, Vincentz M (2008) The role of bZIP transcription factors in green plant evolution: adaptive features emerging from four founder genes. PLoS ONE. 3:116.

Darjani A, Shirani-Rad AH, Gholipour S, Haghighat A (2013) Investigating the effects of water stress on yield and yield components of canola winter varieties. Int J Agron Plant Prod. 4: 370-374.

Diepenbrock W (2000) Yield analysis of winter oilseed rape (Brassica napus L.): a review. Field Crop Res. 67:35-49.

Ding ZJ, Yan JY, Xu XY, Yu DQ, Li GX, Zhang SQ, Zheng SJ (2014) Transcription factor WRKY46 regulates osmotic stress responses and stomatal movement independently in Arabidopsis. Plant J. 79:13-27.

Du L, Chen Z (2000) Identification of genes encoding receptorlike protein kinases as possible targets of pathogen- and salicylic acid-induced WRKY DNA-binding proteins in Arabidopsis. Plant J. 24:837-847.

Eulgem T, Rushton PJ, Robatzek S, Somssich IE (2000) The WRKY superfamily of plant transcription factors. Trends Plant Sci. 5:199-206.

Eulgem T, Somssich IE (2007) Networks of WRKY transcription factors in defense signaling. Curr Opin Plant Biol. 10:366371.

Fan Q, Song A, Xin J, Chen S, Jiang J, Wang Y, Li X, Chen F (2015) CmWRKY15 facilitates Alternaria tenuissima infection of Chrysanthemum. PLoS One. 10:1-18.

Finn RD, Coggill P, Eberhardt RY, Eddy SR, Mistry J, Mitchell AL, Potter SC, Punta M, Qureshi M, Sangrador-Vegas A, Salazar GA, Tate JA, Bateman A (2016) The Pfam protein families database: towards a more sustainable future. Nucleic Acids Res. 44:279-285. 
Froger A, Hall JE (2007) Transformation of plasmid DNA into $E$. coli Using the heat shock method. J Vis Exp. 253:1-1.

Grove MD, Spencer GF, Rohwedder WK, Mandava N, Worley JF, Warthen JD, Steffens GL, Flippen-Anderson JL, Cook JC (1979) Brassinolide, a plant growth-promoting steroid isolated from Brassica napus pollen. Nature. 281:216-217.

He GH, Xu JY, Wang YX, Liu JM, Li PS, Chen M, Ma YZ, Xu ZS (2016) Drought-responsive WRKY transcription factor genes TaWRKY1 and TaWRKY33 from wheat confer drought and/or heat resistance in Arabidopsis. BMC Plant Biology. 116:1-16.

He Y, Mao S, Gao Y, Zhu L, Wu D, Cui Y, Li J, Qian W (2016) Genome-wide identification and expression analysis of WRKY transcription factors under multiple stresses in Brassica napus. PLoS ONE. 11:1-18.

Hinderhofer K, Zentgraf $U$ (2001) Identification of a transcription factor specifically expressed at the onset of leaf senescence. Planta. 213:469-73.

Huang X, Li K, Xu X, Yao Z, Jin C, Zhang S (2015) Genome-wide analysis of WRKY transcription factors in white pear (Pyrus bretschneideri) reveals evolution and patterns under drought stress. BMC Genomics. 16:1-14.

Jiang Y, Liang G, Yu D (2012) Activated expression of WRKY57 confers drought tolerance in arabidopsis. Mol Plant. 5:13751388.

Jiang Y, Liang G, Yang SH, Yu D (2014) Arabidopsis WRKY57 functions as a node of convergence for jasmonic acid and auxin-mediated signaling in jasmonic acid-induced leaf senescence. The Plant Cell. 26:230-245.

Jiang Y, Qiu Y, Hu Y, Yu D (2016) Heterologous expression of AtWRKY57 confers drought tolerance in Oryza sativa. Front Plant Sci. 7:1-11.

Jiang Y, Yu D (2016) WRKY57 regulates JAZ genes transcriptionally to compromise Botrytis cinerea resistance in Arabidopsis thaliana. Plant Physiol. 171:2771-2782.

Johnson CS, Kolevski B, Smyth DR (2002) TRANSPARENT TESTA GLBRA2, a trichome and seed coat development gene of Arabidopsis, encodes a WRKY transcription factor. Plant Cell. 14:1359-75.

Kermode AR (2005) Role of abscisic acid in seed dormancy. J Plant Growth Regul. 24:319-344.

Kumar S, Stecher G, Tamura K (2016) MEGA7: molecular evolutionary genetics analysis version 7.0 for bigger Datasets. Mol Biol Evol. 33:1870-1874.

Kyte J, Doolittle RF (1982) A simple method for displaying the hydropathic character of a protein. J Mol Biol. 157:105-132.

Lagacé M, Matton DP (2004) Characterization of a WRKY transcription factor expressed in late torpedo-stage embryos of Solanum chacoense. Planta. 21:185-9.

Lescot $M$, Déhais $P$, Moreau $Y$, De Moor $B$, Rouzé $P$, Rombauts $S$ (2002) PlantCARE: a database of plant cis-acting regulatory elements and a portal to tools for in silico analysis of promoter sequences. Nucleic Acids Res. 30:325-327.

Liu QL, Zhong M, Li S, Pan YZ, Jiang BB, Jia Y, Zhang HQ (2013) Overexpression of a chrysanthemum transcription factor gene, $D g W R K Y 3$, in tobacco enhances tolerance to salt stress. Plant Physiol Biochem. 69:27-33.
Liu QL, Xu KD, Pan YZ, Jiang BB, Liu GL, Jia Y, Zhang HQ (2014) Functional analysis of a novel chrysanthemum WRKY transcription factor gene involved in salt tolerance. Plant Mol Biol Rep. 32:282-289.

Liu X, Song Y, Xing F, Wang N, Wen F, Zhu C (2016) GhWRKY25, a group I WRKY gene from cotton, confers differential tolerance to abiotic and biotic stresses in transgenic Nicotiana benthamiana. Protoplasma. 253:1265-1281.

Livak KJ, Schmittgen TD (2001) Analysis of relative gene expression data using real time quantitative PCR and the 2 ${ }_{\mathrm{DDC}}$ method. Methods. 25:402-408.

Luo M, Dennis ES, Berger F, Peacock WJ, Chaudhury A (2005) MINISEED3 (MINI3), a WRKY family gene, and HAIKU2 (IKU2), a leucine-rich repeat (LRR) KINASE gene, are regulators of seed size in Arabidopsis. PNAS. 102:17531-6.

Luo X, Bai X, Sun X, Zhu D, Liu B, Ji W, Cai H, Cao L, Wu J, Hu M, Liu X, Tang L, Zhu Y (2013) Expression of wild soybean WRKY20 in Arabidopsis enhances drought tolerance and regulates ABA signalling. J Exp Bot. 64:2155-69.

Ma T, Li M, Zhao A, Xu X, Liu G, Cheng L (2014) LcWRKY5: an unknown function gene from sheepgrass improves drought tolerance in transgenic Arabidopsis. Plant Cell Rep. 33:15071518.

Mirzaee M, Moieni A, Ghanati F (2013) Effects of Drought Stress on the Lipid Peroxidation and Antioxidant Enzyme Activities in Two Canola (Brassica napus L.) Cultivars. J Agr Sci Tech. 15:593-602.

Niu CF, Wei W, Zhou QY, Tian AG, Hao YJ, Zhang WK, Ma B, Lin Q, Zhang ZB, Zhang JS, Chen SY (2012) Wheat WRKY genes TaWRKY2 and TaWRKY19 regulate abiotic stress tolerance in transgenic Arabidopsis plants. Plant Cell Environ. 35:11561170.

Pandey SP, Somssich IE (2009) The role of WRKY transcription factors in plant immunity. Plant Physiol. 150:1648-1655.

Pnueli L, Hallak-Herr E, Rozenberg M, Cohen M, Goloubinoff $P$, Kaplan A (2002) Molecular and biochemical mechanisms associated with dormancy and drought tolerance in the desert legume Retama raetam. Plant J. 31:319-30.

Qiu Y, Yu D (2009) Over-expression of the stress-induced OsWRKY45 enhances disease resistance and drought tolerance in Arabidopsis. Environ Exper Bot. 65:35-47.

Rameeh V, Cherati A, Abbaszadeh F (2012) Salinity effects on yield, yield components and nutrient ions in Rapeseed genotypes. J Agric Sci. 57:19-29.

Raymer PL (2002) Canola: an emerging oilseed crop. Trends New Crops New Uses. 1:122-126.

Rana D, Boogaart TVD, O' Neill CM, Hynes L, Bent E, Macpherson L, Par JY, Lim YP, Bancroft L (2004) Conservation of the microstructure of genome segments in Brassica napus and its diploid relatives. Plant J. 40:725-733.

Ren $X$, Chen Z, Liu $Y$, Zhang $H$, Zhang $M$, Liu $Q$, Hong X, Zhu JK, Gong Z (2010) ABO3, a WRKY transcription factor, mediates plant responses to abscisic acid and drought tolerance in Arabidopsis. The Plant J. 63:417-429.

Rinerson Cl, Rabara RC, Tripathi P, Shen QJ, Rushton PJ (2015) The evolution of WRKY transcription factors. BMC Plant Biol. 15:1-18.

Rushton PJ, Somssich IE, Ringler P, Shen QJ (2010) WRKY transcription factors. Trends Plant Sci. 15:247-258. 
Rushton DL, Tripathi P, Rabara RC, Lin J, Ringler P, Boken AK, Langum TJ, Smidt L, Boomsma DD, Emme NJ, Chen X, Finer JJ, Shen QJ, Rushton PJ (2012) WRKY transcription factors: key components in abscisic acid signaling. Plant Biotechnol J. 10: 2-11.

Ryu H, Cho YG (2015) Plant hormones in salt stress tolerance. J Plant Biol. 58:147-155.

Seki M, Umezawa T, Urano K, Shinozaki K (2007) Regulatory metabolic networks in drought stress responses. Curr Opin Plant Biol. 10:296-302.

Shamloo-Dashtpagerdi R, Razi H, Niazi A, Ebrahimie E (2018) Molecular characterization of Brassica napus stress related transcription factors, BnMYB44 and BnVIP1, selected based on comparative analysis of Arabidopsis thaliana and Eutrema salsugineum transcriptomes. Mol Biol Rep. 45: 1111-1124.

Xie FL, Huang SQ, Guo K, Xiang AL, Zhu YY, Nie L, Yang ZM (2007) Computational identification of novel microRNAs and targets in Brassica napus. FEBS Lett. 581:1464-1474.

Yamaguchi-Shinozaki K, Shinozaki K (2006) Transcriptional regulatory networks in cellular responses and tolerance to dehydration and cold stresses. Annu Rev Plant Biol. 57: 781-803.

Yang B, Jiang Y, Rahman MH, Deyholos MK, Kav NN (2009) Identification and expression analysis of WRKY transcription factor genes in canola (Brassica napus L.) in response to fungal pathogens and hormone treatments. BMC Plant Biol. 9:1-19.
Yang SJ, Vanderbeld B, Wan JX, Huang YF (2010) Narrowing down the targets: toward successful genetic engineering of drought-tolerant crops. Mol Plant. 3:469-90.

Zhang ZL, Xie Z, Zou X, Casaretto J, Ho TH, Shen QJ (2004) A rice WRKY gene encodes a transcriptional repressor of the gibberellin signaling pathway in aleurone cells. Plant Physiology. 134:1500-1513.

Zhao JL, Wang YL, Yao DQ, Zhu WY, Chen L, He HL, Pan JS, Cai R (2015) Transcriptome profiling of trichome-less reveals genes associated with multicellular trichome development in Cucumis sativus. Mol Genet Genomics. 290:2007-18.

Zhou QY, Tian AG, Zou HF, Xie ZM, Lei G, Huang J (2008) Soybean WRKY type transcription factor genes, GmWRKY13, GmWRKY21, and GmWRKY54, confer differential tolerance to abiotic stresses in transgenic Arabidopsis plants. Plant Biotechnol. 6:486-503. 